ALEA, Lat. Am. J. Probab. Math. Stat. 13, 1151-1164 (2016)

DOI: 10.30757 /ALEA.v13-41

\title{
The limiting shape of a full mailbox
}

\section{Marco Formentin and Jan M. Swart}

Institute of Information Theory and Automation of the ASCR, Pod Vodárenskou věží 4, CZ-18208 Prague, Czech Republic

E-mail address: marco.formentin@rub.de

Institute of Information Theory and Automation of the ASCR, Pod Vodárenskou věží 4, CZ-18208 Prague, Czech Republic

E-mail address: swart@utia.cas.cz

URL: http://staff.utia.cas.cz/swart/

\begin{abstract}
We study a model for email communication due to Gabrielli and Caldarelli, where someone receives and answers emails at the times of independent Poisson processes with intensities $\lambda_{\text {in }}>\lambda_{\text {out }}$. The receiver assigns i.i.d. priorities to incoming emails according to some atomless law and always answers the email in the mailbox with the highest priority. Since the frequency of incoming emails is higher than the frequency of answering, below a critical priority, the mailbox fills up ad infinitum. We prove a theorem about the limiting shape of the mailbox just above the critical point, linking it to the convex hull of Brownian motion. We conjecture that this limiting shape is universal in a class of similar models, including a model for the evolution of an order book due to Stigler and Luckock.
\end{abstract}

\section{Introduction}

1.1. Description of the model. The queueing model we study in this paper was introduced by Gabrielli and Caldarelli (2009) as a variation of Barabási's queueing system Barabasi (2005). These and similar models have been investigated in recent years in the complex system literature as they are able to capture some universal patterns in human written communication Blanchard and Hongler (2007); Formentin et al. (2014); Vázquez et al. (2006). Usually in this context, the model aims to describe the response time statistics of a user which assigns a subjective priority to each incoming message (email, paper mail or sms) and then answers first the highest priority one. We will be interested in a somewhat different function of the process, namely, the asymptotics of the number of items waiting to be executed

Received by the editors November 17, 2015; accepted November 11, 2016.

2010 Mathematics Subject Classification. 82C27, 60K35, 82C26, 60K25.

Key words and phrases. Self-organized criticality, Gabrielli and Caldarelli queueing model, Barabasi queueing model, email communication. 
with priority close to the critical point. The mathematical description of the model is as follows.

Tasks arrive according to a Poisson point process with rate $\lambda_{\text {in }}$. Each incoming task is assigned a priority. The priorities of incoming tasks are i.i.d. real-valued random variables with some law $\mu$. We assume that $\mu$ is atomless, which assures that all tasks in the queue have a different priority. At times of a Poisson point process with rate $\lambda_{\text {out }}$, the task with the highest priority in the queue is executed (i.e., removed from the queue). If at such a time, the queue is empty, then nothing happens.

Since only the relative order of the priorities matters, the precise choice of the law $\mu$ does not matter. For definiteness, we choose for $\mu$ the uniform distribution on $\left[-\lambda_{\text {in }}, 0\right]$. Note that our priorities are negative numbers, i.e., 0 is the highest possible priority, which will be convenient from a mathematical perspective. By time scaling, we can without loss of generality assume that $\lambda_{\text {out }}=1$ so that our model depends on a single parameter $\lambda:=\lambda_{\text {in }}$.

Let $\Pi_{\mathrm{in}}^{\lambda}$ be the random collection of all pairs $(p, t)$, where $t \geq 0$ is a time when a new task arrives and $p \in[-\lambda, 0]$ is the priority assigned to this task. Also, let $\Pi_{\text {out }}$ be the collection of all times $s \geq 0$ when tasks are executed. Let $Y^{\lambda}(t)$ be a finite subset of $[-\lambda, 0]$, describing the priorities of all tasks in the queue at time $t$. Since all tasks have a different priority, the cardinality $N^{\lambda}(t):=\left|Y^{\lambda}(t)\right|$ of $Y^{\lambda}(t)$ equals the number of tasks in the queue. By convention, we choose $Y^{\lambda}(t)$ right-continuous in $t$ and let $Y^{\lambda}(t-):=\lim _{s \uparrow t} Y^{\lambda}(s)$ denote the state of $Y^{\lambda}$ immediately prior to $t$. We order the elements of $Y^{\lambda}(t)$ from the highest to the lowest priority:

$$
Y^{\lambda}(t)=\left\{Y_{1}^{\lambda}(t), \ldots, Y_{N^{\lambda}(t)}^{\lambda}\right\} \quad \text { with } \quad Y_{1}^{\lambda}(t)>Y_{2}^{\lambda}(t)>\cdots>Y_{N^{\lambda}(t)}^{\lambda}(t) .
$$

We start the process with $Y^{\lambda}(0)$ some finite subset of $[-\lambda, 0]$. Then $\left(Y^{\lambda}(t)\right)_{t \geq 0}$ is a continuous-time Markov process with the following description:

(i) For each $(p, t) \in \Pi_{\text {in }}^{\lambda}$, at time $t$, the previous state $Y^{\lambda}(t-)$ of the process is replaced by $Y^{\lambda}(t):=Y^{\lambda}(t-) \cup\{p\}$.

(ii) For each $t \in \Pi_{\text {out }}$, at time $t$, the previous state $Y^{\lambda}(t-)$ of the process is replaced by $Y^{\lambda}(t):=Y^{\lambda}(t-) \backslash\left\{Y_{1}^{\lambda}(t-)\right\}$ if $Y^{\lambda}(t-) \neq \emptyset$, and stays empty otherwise.

We call $Y^{\lambda}=\left(Y^{\lambda}(t)\right)_{t \geq 0}$ the inbox process with rate of incoming tasks $\lambda \geq 0$.

We observe that $\Pi_{\text {in }}^{\lambda}$ is a Poisson process on $[-\lambda, 0] \times[0, \infty)$ with intensity one. It will be convenient to construct $\Pi_{\text {in }}^{\lambda}$ in the following way: letting $\Pi_{\text {in }}$ denote a Poisson process on $(-\infty, 0] \times[0, \infty)$ with intensity one, we define $\Pi_{\mathrm{in}}^{\lambda}$ as the restriction

$$
\Pi_{\text {in }}^{\lambda}:=\Pi_{\text {in }} \cap([-\lambda, 0] \times[0, \infty)) .
$$

We observe the following consistency relation. If $0 \leq \lambda^{\prime} \leq \lambda$, then setting

$$
Y^{\lambda^{\prime}}(t):=Y^{\lambda}(t) \cap\left[-\lambda^{\prime}, 0\right] \quad(t \geq 0)
$$

is exactly the inbox process with rate of incoming tasks $\lambda^{\prime}$, started in the initial state $Y^{\lambda^{\prime}}(0):=Y^{\lambda}(0) \cap\left[-\lambda^{\prime}, 0\right]$ and constructed from the Poisson processes $\Pi_{\mathrm{in}}^{\lambda^{\prime}}$ and $\Pi_{\text {out }}$. Indeed, incoming tasks with a priority below $-\lambda^{\prime}$ have no influence on $Y^{\lambda^{\prime}}$. Also, at times when a task with priority below $-\lambda^{\prime}$ is executed, the random set $Y^{\lambda^{\prime}}(t)$ is empty and stays empty, in line with the rules above. 
In view of this, we can remove the last free parameter of our model and, starting from a locally finite ${ }^{1}$ subset $Y(0) \subset(-\infty, 0]$, define an "infinite" process $(Y(t))_{t \geq 0}$ taking values in the locally finite subsets of $(-\infty, 0]$ such that for each $\lambda \geq 0$,

$$
Y^{\lambda}(t)=Y(t) \cap[-\lambda, 0] \quad(t \geq 0)
$$

is the inbox process with rate of incoming tasks $\lambda$, started in the initial state $Y^{\lambda}(0):=Y(0) \cap[-\lambda, 0]$. Formally, the process $Y$ follows the same rules as $Y^{\lambda}$, with $\Pi_{\text {in }}^{\lambda}$ replaced by $\Pi_{\text {in }}$. Because of consistency, $Y$ is well-defined, even though the set of times $\left\{t \geq 0:(p, t) \in \Pi_{\text {in }}\right\}$ is a.s. dense in $[0, \infty)$.

1.2. The critical point. Recall that $N^{\lambda}(t)$ denotes the number of tasks in the queue with priority in $[-\lambda, 0]$. We observe that $N^{\lambda}=\left(N^{\lambda}(t)\right)_{t>0}$ is a continuous-time random walk with reflection at the origin, i.e., $N^{\lambda}$ is a Markov process with state space $\mathbb{N}$ that jumps

$$
n \mapsto n+1 \text { with rate } \lambda \text { and } n \mapsto n-1 \text { with rate } 1_{\{n>0\}} \text {. }
$$

This process is positive recurrent for $\lambda<1$, null recurrent for $\lambda=1$, and transient for $\lambda>1$. We order the elements of $Y(t)$ as $Y_{1}(t)>Y_{2}(t)>\cdots$ as in (1.1). Transience for $\lambda>1$ and recurrence for $\lambda \leq 1$ imply that

$$
\liminf _{t \rightarrow \infty} Y_{1}(t)=-1 \quad \text { a.s. }
$$

so for each $\lambda>1$, there is a random time after which no tasks with a priority below $-\lambda$ are executed anymore. On the other hand, for each $\lambda<1$, positive recurrence implies that the highest priority $Y_{1}(t)$ in the inbox spends a positive fraction of time below $-\lambda$. In view of this, the following proposition should not come as a surprise. Recall that a subset of a topological space is locally finite if its intersection with any compact set is finite. In particular, a subset $Y \subset(-1,0]$ is locally finite if $Y \cap[-\lambda, 1]$ is finite for all $0<\lambda<1$.

Proposition 1.1 (Long-time limit). There exists a random, locally finite subset $Y^{1}(\infty) \subset(-1,0]$ such that, regardless of the initial state $Y(0)$,

$$
\mathbb{P}\left[Y^{\lambda}(t) \in \cdot\right] \underset{t \rightarrow \infty}{\longrightarrow} \mathbb{P}\left[Y^{1}(\infty) \cap[-\lambda, 0] \in \cdot\right] \quad(0<\lambda<1)
$$

where $\rightarrow$ denotes convergence of probability measures in total variation norm distance. The random point set $Y^{1}(\infty)$ a.s. has infinitely many elements. Writing

$$
Y^{\lambda}(\infty):=Y^{1}(\infty) \cap[-\lambda, 0] \quad \text { and } \quad N^{\lambda}(\infty):=\left|Y^{\lambda}(\infty)\right| \quad(0 \leq \lambda \leq 1),
$$

one has

$$
\mathbb{P}\left[N^{\lambda}(\infty)=n\right]=(1-\lambda) \lambda^{n} \quad(0 \leq \lambda<1, n \geq 0)
$$

The random set $Y^{1}(\infty)$ describes the long-time limit of the collection of all tasks in the inbox with priorities above the critical point $-\lambda_{\mathrm{c}}:=-1$ waiting to be executed. We are interested in the shape of $Y^{1}(\infty)$ near the critical point.

\footnotetext{
${ }^{1}$ By definition, a subset $Y$ of a topological space $X$ is locally finite if $Y \cap C$ is finite for each compact subset $C$ of $X$.
} 
1.3. The limiting shape near the critical point. Recall from Proposition 1.1 that $N^{\lambda}(\infty)$ denotes the equilibrium number of tasks with priority $>-\lambda$ in the inbox, which is a.s. finite by the local finiteness of $Y^{1}(\infty) \subset(-1,0]$. We will be interested in the shape of the random function $\lambda \mapsto N^{\lambda}(\infty)$ in the vicinity of $\lambda_{\mathrm{c}}=1$. To this aim, for $\varepsilon>0$, we define

$$
H_{s}^{\varepsilon}:=\varepsilon N^{1-2 \varepsilon s}(\infty) \quad(s>0) .
$$

Clearly, $H^{\varepsilon}:(0, \infty) \rightarrow[0, \infty)$ is a.s. right-continuous, nonincreasing, $H^{\varepsilon}(s)=0$ for $s \geq 1 /(2 \varepsilon)$, and $\lim _{s \downarrow 0} H^{\varepsilon}(s)=\infty$ by the fact (proved in Proposition 1.1) that $\left|Y^{1}(\infty)\right|=\infty$ a.s. As $\varepsilon \downarrow 0$, the function $H^{\varepsilon}$ describes the shape of the function $\lambda \mapsto N^{\lambda}(\infty)$ for $\lambda$ just below $\lambda_{\mathrm{c}}=1$, where we scale distances in $\lambda$ by a factor $(2 \varepsilon)^{-1}$ and at the same time scale down the numbers $N^{\lambda}(\infty)$ by giving each task a weight $\varepsilon$.

We will prove that the random function $H^{\varepsilon}$ converges as $\varepsilon \downarrow 0$ to a random limiting function, that is closely linked to the convex hull of Brownian motion. To formulate this properly, let $\mathcal{H}$ denote the space of all right-continuous, nonincreasing functions $h:(0, \infty) \rightarrow[0, \infty)$. Then $h \in \mathcal{H}$ if and only if $h$ is the distribution function of a locally finite measure on $(0, \infty]$, i.e., each $h \in \mathcal{H}$ corresponds to a locally finite measure $\mu$ on $(0, \infty]$ such that

$$
h_{s}=\mu((s, \infty]) \quad(s \in(0, \infty)) \text {. }
$$

We equip $\mathcal{H}$ with the topology of vague convergence of the corresponding locally finite measures on $(0, \infty]$. The following theorem is our main result.

Theorem 1.2 (Limiting shape near the critical point). One has

$$
\mathbb{P}\left[\left(H_{s}^{\varepsilon}\right)_{s>0} \in \cdot\right] \underset{\varepsilon \downarrow 0}{\Longrightarrow} \mathbb{P}\left[\left(H_{s}\right)_{s>0} \in \cdot\right]
$$

where $\Rightarrow$ denotes weak convergence of probability laws on $\mathcal{H}$ with respect to the topology defined above, and $H_{s}:=\sup _{t \geq 0}\left(B_{t}-s t\right)$ with $\left(B_{t}\right)_{t \geq 0}$ standard Brownian motion.

It is known that the function $\left(H_{s}\right)_{s>0}$ of Theorem 1.2 is piecewise linear. Let $H_{s}^{\prime}:=\frac{\partial}{\partial s} H_{s}$ denote the left derivative of $H_{s}$ and set $\tau(a):=-H_{1 / a}^{\prime}(a>0)$ with $\tau(0):=0$. In Groeneboom (1983), it is proved that $(\tau(a))_{a \geq 0}$ is a jump process with independent non-stationary increments. Moreover, its number of jumps in an interval $(a, b)$ with $0<a<b<\infty$ is Poisson distributed with mean $\log (b / a)$. Note that this implies that for $0<a<b<\infty$, the number of points in $(a, b)$ where the derivative of $H_{s}$ makes a jump is also Poisson distributed with mean $\log (b / a)$. See Figure 1.1 for a typical trajectory of $\left(H_{s}\right)_{s>0}$ and an explanation of the fact that $-H_{s}^{\prime}=\inf \left\{t \geq 0: B_{t}-s t=H_{s}\right\}(s>0)$.

In our model, the quantity $-\frac{\partial}{\partial s} H_{s}$ describes the local Poisson density of tasks with priority close to $s$ and hence is proportional to the time that has passed since the last time that the highest-priori task $Y_{1}$ in the queue had a value below $s$. A jump of $-\frac{\partial}{\partial s} H_{s}$ at some priority $s_{\star}$ reflects the fact that at some quite distant moment in the past, $Y_{1}$, coming from the right, reached a local minimum at $s_{\star}$ before moving up again. The relation between jumps of $-\frac{\partial}{\partial s} H_{s}$ and the convex hull of Brownian motion is explained in Figure 1.2. 

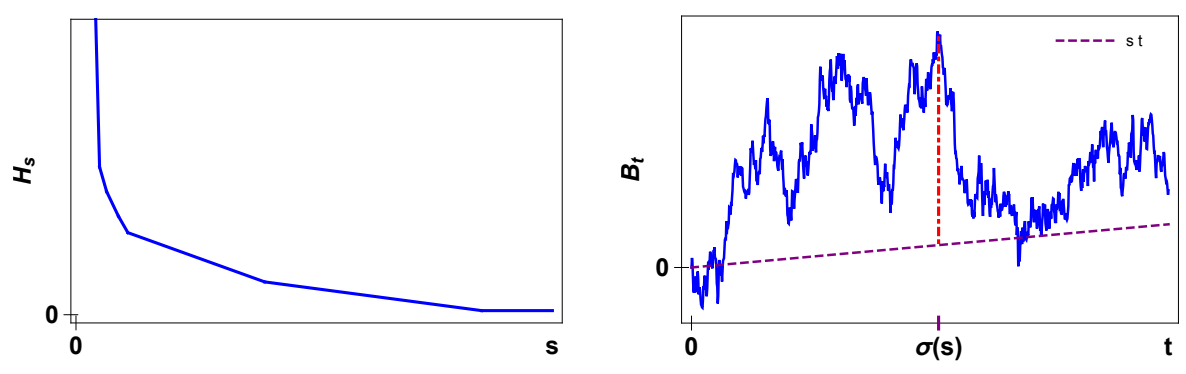

Figure 1.1. Simulation showing a typical trajectory of $\left(H_{s}\right)_{s \geq 0}$ (left panel). Letting $\sigma(s):=\inf \left\{t \geq 0: B_{t}-s t=H_{s}\right\}$ be the position where $B_{t}-s t$ assumes its maximum, we observe that $H_{s}=B_{\sigma(s)}-\sigma(s) s$ and hence $-\frac{\partial}{\partial s} H_{s}=\sigma(s)$.
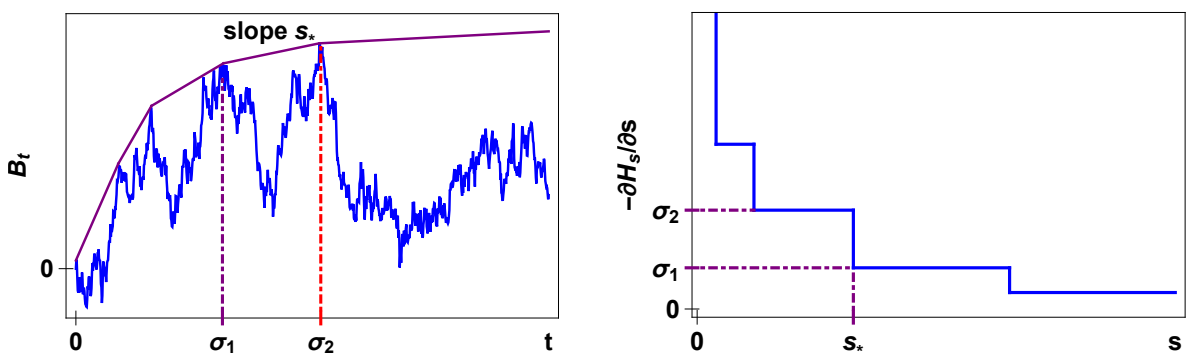

Figure 1.2. Relation of $H_{s}$ to the convex hull of Brownian motion (on the left). The slope $s_{\star}$ of a line segment of the convex hull corresponds to a value of $s$ where the position $\sigma(s)$ of the maximum of $B_{t}-s t$ makes a jump, which corresponds to a jump of $-\frac{\partial}{\partial s} H_{s}$ (see Figure 1.1).

1.4. Discussion and overview. The inbox model of Gabrielli and Caldarelli (2009) that is our object of study in the present paper exhibits self-organized criticality. Indeed, the model organizes itself in such a way that critical behavior associated with the transition between recurrence and transience can be observed due to incoming tasks with a priority close to the critical point $\lambda_{\mathrm{c}}=1$. This expresses itself in a power law for serving times as demonstated in Gabrielli and Caldarelli (2009) and also in our main result Theorem 1.2 that shows that in equilibrium, the number of tasks with priority above $-\lambda$ is of order $\left(\lambda_{\mathrm{c}}-\lambda\right)^{-1}$.

Gabrielli and Caldarelli's model is reminiscent of the well-known Bak and Sneppen (1993) model, which is one of the best-known models exhibiting self-organized criticality, although this is only been fully rigorously established for a simplified version of the model Meester and Sarkar (2012). Other similar models are a "onesided canyon model" introduced in Swart (2015) and a model for traders placing limit buy and sell limit orders at a stock market, first introduced by Stigler (1964) and, in a more general form, by Luckock (2003). All these models are based on a variation of the rule "kill the highest (or lowest) particle", and all these models exhibit a steady state where particles cluster near a critical point. We also mention 
one-dimensional spatial branching processes where at each branching event, the lowest particles are killed to keep the number of particles constant. These models also exhibit self-organized criticality. They have been introduced with a biological interpretation in Brunet et al. (2006) and have since also been studied in the mathematical literature in e.g. Maillard (2016).

It is interesting to speculate to what degree our main result Theorem 1.2 describes universal behavior in this class of models. We expect the statement to be true for the steady states of the models in Swart (2015) and Luckock (2003), and possibly (in a somewhat modified form) for the model in Meester and Sarkar (2012). Proving this will be considerably more difficult than for the present model, however. A great simplifying property of the inbox model is that the number of tasks $N^{\lambda}(t)$ above a fixed priority is a Markov process, and the same is true for the restriction of $Y(t)$ to $[-\lambda, 0]$. For the model in Swart (2015), this first property fails but the second is still true; for the model in Luckock (2003), both fail.

As an open problem for the inbox model, we mention the following. In our main result Theorem 1.2, we rescale space and the weight of items, but we look only at one fixed time. Is it possible to rescale also time and obtain a Markov process taking values in the space $\mathcal{H}$ that has the law on the right-hand side of (1.12) as its invariant law?

The rest of the paper is devoted to proofs. After some initial observations and definitions in Subsection 2.1, we prove our main result (Theorem 1.2) in Subsection 2.2. The proof depends on some lemmas that are proved in Subsection 2.3. The paper concludes with the proof of Proposition 1.1 in Subsection 2.4.

\section{Proofs}

2.1. The lower invariant process. Extend the Poisson point sets $\Pi_{\text {in }}$ and $\Pi_{\text {out }}$ to negative times, i.e., let $\Pi_{\text {in }}$ be a Poisson point set on $(-\infty, 0] \times \mathbb{R}$ with intensity one and $\Pi_{\text {out }}$ a Poisson process on $\mathbb{R}$ with intensity 1 . For each starting time $s \in \mathbb{R}$ and locally finite subset $y \subset(-\infty, 0]$, we set

$$
\mathbf{Y}_{s, t}(y):=Y(t) \quad(t \geq s)
$$

where $(Y(t))_{t \geq s}$ is the inbox process started at time $s$ in the initial state $Y(s)=y$ and defined in terms of $\Pi_{\text {in }}$ and $\Pi_{\text {out }}$ as in Section 1.1. Then $\left(\mathbf{Y}_{s, t}\right)_{s \leq t}$ is a stochastic flow, i.e., a collection of random maps such that $\mathbf{Y}_{s, s}$ is the identity map and $\mathbf{Y}_{t, u} \circ \mathbf{Y}_{s, t}=\mathbf{Y}_{s, u}$, almost surely for all $s \leq t \leq u$. The next lemma says that these maps are monotone with respect to set inclusion.

Lemma 2.1 (Monotonicity). Almost surely, $y \subset \tilde{y}$ implies $\mathbf{Y}_{s, t}(y) \subset \mathbf{Y}_{s, t}(\tilde{y})$ for all $s \leq t$ and locally finite subsets $y, \tilde{y} \subset(-\infty, 0]$.

Proof: Let $Y(t):=\mathbf{Y}_{s, t}(y)$ and $\tilde{Y}(t):=\mathbf{Y}_{s, t}(\tilde{y})$. If $t$ is a time when an incoming task of priority $\lambda$ arrives, then this task is added both to $Y$ and $\tilde{Y}$, so $Y(t-) \subset$ $\tilde{Y}(t-)$ implies $Y(t) \subset \tilde{Y}(t)$. If $t$ is a time when a task is executed, then the task with the highest priority (if there is one) is removed from both to $Y$ and $\tilde{Y}$. If $Y(t-) \subset \tilde{Y}(t-)$, then either the highest priority element $\tilde{Y}_{1}(t-)$ is not an element of $Y(t-)$, or $\tilde{Y}_{1}(t-)=Y_{1}(t-)$, so also in this case the inclusion is preserved. 
The construction of the stationary process $(\underline{Y}(t))_{t \in \mathbb{R}}$ from the next lemma is similar to the construction of the lower invariant law of a monotone interacting particle system (see Liggett, 2005, Thm III.2.3).

Lemma 2.2 (Lower invariant process). Almost surely, for each $t \in \mathbb{R}$ there exists a random countable subset $\underline{Y}(t) \subset(-\infty, 0]$ such that

$$
\mathbf{Y}_{s, t}(\emptyset) \uparrow \underline{Y}(t) \quad \text { as } s \downarrow-\infty .
$$

Proof: For each $s \leq s^{\prime}$, one has $\mathbf{Y}_{s^{\prime}, s^{\prime}}(\emptyset)=\emptyset \subset \mathbf{Y}_{s, s^{\prime}}(\emptyset)$. Using the stochastic flow property and Lemma 2.1, we see that $\mathbf{Y}_{s^{\prime}, t}(\emptyset) \subset \mathbf{Y}_{s, t}(\emptyset)$ for all $s \leq s^{\prime} \leq t$. It follows that the left-hand side of (2.2) increases to a limit as $s \downarrow-\infty$.

In line with earlier notation, we denote

$$
\underline{Y^{\lambda}}(t):=\underline{Y}(t) \cap[-\lambda, 0] \quad \text { and } \quad \underline{N}^{\lambda}(t):=\left|\underline{Y}^{\lambda}(t)\right| \quad(t \in \mathbb{R}, \lambda \geq 0) .
$$

For $0 \leq \delta \leq 1$, we will derive a formula for $\underline{N}^{1-\delta}(0)$ that is reminiscent of the definition of the process $H_{s}$ from Theorem 1.2. As a first step, we prove the following simple lemma.

Lemma 2.3 (Reflected random walk). For $0 \leq \delta \leq 1$ and $u \leq 0$, let

$$
E^{\delta}(u):=\left|\Pi_{\text {out }} \cap[u, 0]\right|-\left|\Pi_{\text {in }} \cap([-1+\delta, 0] \times[u, 0])\right|
$$

denote the number of times that a task is executed in the time interval $[u, 0]$ minus the number of tasks with priority $\geq-(1-\delta)$ that arrive in the time interval $[u, 0]$. Then

$$
\left|\mathbf{Y}_{s, u}(\emptyset) \cap[-1+\delta, 0]\right|=E^{\delta}(u)-\inf _{s \leq t \leq u} E^{\delta}(t) \quad(s \leq u \leq 0)
$$

Proof: Clearly, the left- and right-hand sides of (2.5) are both zero if $s=u$. Increasing $u$ for fixed $s$, we observe that both sides of (2.5) increase by one if a task arrives with priority in $[-(1-\delta), 1]$. At times of $\Pi_{\text {out }}$, either both sides of $(2.5)$ are zero and remain zero (due to the fact that both $E^{\delta}(u)$ and its running infimum decrease by one), or both sides of (2.5) are nonzero and decrease by one.

Setting $u=0$ in (2.5) and letting $s \downarrow-\infty$, using the fact that $E^{\delta}(0)=0$, it follows that

$$
\underline{N}^{1-\delta}(0)=-\inf _{t \leq 0} E^{\delta}(t) .
$$

In view of what follows, it will be convenient to write the random walk $E^{\delta}$ as the sum of a driftless random walk (which will converge to Brownian motion) and a term that contains the drift (which will converge to a linear function). To this aim, we define, for each $t \geq 0$,

$$
F(t):=\left|\Pi_{\text {in }} \cap([-1,0] \times[-t, 0])\right|-\left|\Pi_{\text {out }} \cap[-t, 0]\right| \quad(t \geq 0) .
$$

In words, $F(t)$ is the number of tasks with priority $\geq-1$ that arrive in the time interval $[-t, 0]$ minus the number of times that a task is executed in the time interval $[-t, 0]$. Next, for $0 \leq \delta \leq 1$ and $t \geq 0$, we define

$$
G^{\delta}(t):=\left|\Pi_{\text {in }} \cap([-1,-1+\delta] \times[-t, 0])\right| \quad(t \geq 0),
$$

which is the number of tasks with priority in $[-1,-(1-\delta)]$ that arrive in the time interval $[-t, 0]$. 
Proposition 2.4 (Supremum formula). Almost surely, for all $0 \leq \delta \leq 1$,

$$
\underline{N}^{1-\delta}(0)=\sup _{t \geq 0}\left(F(t)-G^{\delta}(t)\right) .
$$

Proof: Since $F(t)-G^{\delta}(t)=-E^{\delta}(-t)(t \geq 0)$, formula (2.9) is just a rewrite of (2.6).

2.2. The diffusive scaling limit. In this section, we prove our main result Theorem 1.2. In view of (1.10), we are interested in $\varepsilon \underline{N}^{1-2 \varepsilon s}(0)$, which by Proposition 2.4 is given by

$$
\begin{aligned}
\varepsilon \underline{N}^{1-2 \varepsilon s}(0) & =\sup _{t \geq 0}\left(\varepsilon F(t)-\varepsilon G^{2 \varepsilon s}(t)\right) \\
& =\sup _{t \geq 0}\left(\varepsilon F\left(\frac{1}{2} \varepsilon^{-2} t\right)-\varepsilon G^{2 \varepsilon s}\left(\frac{1}{2} \varepsilon^{-2} t\right)\right) \quad\left(\varepsilon>0,0 \leq s \leq(2 \varepsilon)^{-1}\right) .
\end{aligned}
$$

For each $\varepsilon>0$, we define rescaled functions $F^{(\varepsilon)}:[0, \infty) \rightarrow \mathbb{R}$ and $G^{(\varepsilon)}:[0, \infty)^{2} \rightarrow$ $\mathbb{R}$ by

$$
\left.\begin{array}{rl}
F^{(\varepsilon)}(t) & :=\varepsilon F\left(\frac{1}{2} \varepsilon^{-2} t\right), \\
G^{(\varepsilon)}(s, t) & :=\varepsilon G^{1 \wedge 2 \varepsilon s}\left(\frac{1}{2} \varepsilon^{-2} t\right)
\end{array}\right\} \quad(s, t \geq 0) .
$$

We will show that as $\varepsilon \downarrow 0$, the function $F^{(\varepsilon)}$ approximates Brownian motion and $G^{(\varepsilon)}(s, t)$ approximates st. We need this convergence to be locally uniform in $s$ and $t$. The easiest way to formulate this is to use coupling, i.e., we replace $\left(F^{(\varepsilon)}, G^{(\varepsilon)}\right)$ by random variables defined on a different underlying probability space, but with the same distribution as the old ones, so that the convergence is almost sure.

Lemma 2.5 (Convergence of coupled processes). For each $\varepsilon_{n} \downarrow 0$, it is possible to couple the random variables $\left(F^{\left(\varepsilon_{n}\right)}, G^{\left(\varepsilon_{n}\right)}\right)$ with $n \geq 0$ in such a way, that almost surely

$$
\begin{aligned}
\sup _{t \in[0, T]}\left|F^{\left(\varepsilon_{n}\right)}(t)-B_{t}\right| \underset{n \rightarrow \infty}{\longrightarrow} 0 & \forall T<\infty, \\
\sup _{(s, t) \in[0, S] \times[0, T]}\left|G^{\left(\varepsilon_{n}\right)}(s, t)-s t\right| \underset{n \rightarrow \infty}{\longrightarrow} 0 & \forall S, T<\infty,
\end{aligned}
$$

where $\left(B_{t}\right)_{t \geq 0}$ is a standard Brownian motion.

The second ingredient in the proof of Theorem 1.2 is the following estimate, which guarantees that the supremum over $t \geq 0$ in (2.10) and the limit $\varepsilon \downarrow 0$ can be interchanged.

Lemma 2.6 (Uniform upper estimate). For each $s>0$, one has

$$
\lim _{T \rightarrow \infty} \sup _{\varepsilon \in(0,1]} \mathbb{P}\left[F^{(\varepsilon)}(t)-G^{(\varepsilon)}(s, t) \geq 0 \text { for some } t \geq T\right]=0 .
$$

The final ingredient for the proof of Theorem 1.2 is a convergence criterion for the topology on $\mathcal{H}$ if the limit function is continuous.

Lemma 2.7 (Continuous limit). Let $\mathcal{H}$ be the space of functions defined in Subsection 1.3. Let $h^{n}, h \in \mathcal{H}$ and assume that $h$ is continuous. Then the following statements are equivalent.

(i) $h^{n} \rightarrow h$ in the topology on $\mathcal{H}$.

(ii) $\sup _{s \in\left[s_{0}, \infty\right)}\left|h_{s}^{n}-h_{s}\right| \underset{n \rightarrow \infty}{\longrightarrow} 0$ for all $s_{0}>0$.

(iii) $h_{s}^{n} \underset{n \rightarrow \infty}{\longrightarrow} h_{s}$ for all $s>0$. 
We first show how Lemmas 2.5-2.7 imply Theorem 1.2, and in the next subsection then prove the lemmas.

Proof of Theorem 1.2: Lemma 2.2 proves the convergence in (1.7) in the special case that $Y(0)=\emptyset$, where $Y^{1}(\infty)$ is equal in distribution to $\underline{Y}^{1}(0)$. Pending the proof of Proposition 1.1, we will prove Theorem 1.2 with the definition (1.10) replaced by $H_{s}^{\varepsilon}:=\varepsilon \underline{N}^{1-2 \varepsilon s}(0)$. Then (2.10) tells us that

$$
H_{s}^{\varepsilon}=\sup _{t \geq 0}\left(F^{(\varepsilon)}(t)-G^{(\varepsilon)}(s, t)\right)
$$

where $F^{(\varepsilon)}$ and $G^{(\varepsilon)}$ are defined in (2.11).

We observe that for any $f, g:[0, T] \rightarrow \mathbb{R}$, one has

$$
\left|\sup _{t \in[0, T]} f(t)-\sup _{t \in[0, T]} g(t)\right| \leq \sup _{t \in[0, T]}|f(t)-g(t)|,
$$

i.e., the map that assigns to a function on $[0, T]$ its supremum is continuous with respect to the supremum norm. In view of this, let us write

$$
\left.\begin{array}{l}
H_{T, s}^{\varepsilon}:=\sup _{t \in[0, T]}\left(F^{(\varepsilon)}(t)-G^{(\varepsilon)}(s, t)\right), \\
H_{T, s}:=\sup _{t \in[0, T]}\left(B_{t}-s t\right) .
\end{array}\right\} \quad(T<\infty, s \geq 0, \varepsilon>0) .
$$

Then, for any $\varepsilon_{n} \downarrow 0$, coupling the random variables $\left(F^{\left(\varepsilon_{n}\right)}, G^{\left(\varepsilon_{n}\right)}\right)$ as in Lemma 2.5, we observe that almost surely,

$$
\sup _{s \in[0, S]}\left|H_{T, s}^{\varepsilon_{n}}-H_{T, s}\right| \underset{n \rightarrow \infty}{\longrightarrow} 0 \quad(S, T<\infty) .
$$

Fix $s_{0}>0$. Then

$$
\mathbb{P}\left[B_{t}-s t \geq 0 \text { for some } t \geq T, s \geq s_{0}\right] \leq \mathbb{P}\left[B_{t}-s_{0} t \geq 0 \text { for some } t \geq T\right] \underset{T \rightarrow \infty}{\longrightarrow} 0,
$$

and hence

$$
\mathbb{P}\left[H_{T, s} \neq H_{s} \text { for some } s \geq s_{0}\right] \underset{T \rightarrow \infty}{\longrightarrow} 0 .
$$

Similarly, using the fact that $G^{(\varepsilon)}(s, t)$ is nondecreasing in $s$, we obtain from Lemma 2.6 that

$$
\sup _{\varepsilon \in(0,1]} \mathbb{P}\left[H_{T, s}^{\varepsilon} \neq H_{s}^{\varepsilon} \text { for some } s \geq s_{0}\right] \underset{T \rightarrow \infty}{\longrightarrow} 0 .
$$

Combining this with (2.17), we see that

$$
\mathbb{P}\left[\sup _{s \in\left[s_{0}, S\right]}\left|H_{s}^{\varepsilon_{n}}-H_{s}\right| \geq \delta\right] \underset{n \rightarrow \infty}{\longrightarrow} 0 \quad\left(0<s_{0}<S<\infty, \delta>0\right) .
$$

This implies that

$$
\mathbb{E}\left[1 \wedge \sup _{s \in\left[s_{0}, S\right]}\left|H_{s}^{\varepsilon_{n}}-H_{s}\right|\right] \underset{n \rightarrow \infty}{\longrightarrow} 0 \quad\left(0<s_{0}<S<\infty\right)
$$

and hence also

$$
\mathbb{E}\left[\sum_{N=2}^{\infty} 2^{-N}\left(1 \wedge \sup _{s \in[1 / N, N]}\left|H_{s}^{\varepsilon_{n}}-H_{s}\right|\right)\right] \underset{n \rightarrow \infty}{\longrightarrow} 0 .
$$


It follows that there is a subsequence $\varepsilon_{n(m)}$ such that the expression in the expectation converges to zero a.s. (compare the proof of Kallenberg (1997, Lemma 3.2)), which by Lemma 2.7 implies that

$$
H^{\varepsilon_{n(m)}} \underset{n \rightarrow \infty}{\longrightarrow} H \text { a.s. }
$$

where $\rightarrow$ denotes convergence in the topology on $\mathcal{H}$. Letting $\Rightarrow$ denote weak convergence of probability laws on $\mathcal{H}$ with respect to this topology, it follows that every sequence $\varepsilon_{n} \downarrow 0$ contains a subsequence $\varepsilon_{n(m)}$ such that

$$
\mathbb{P}\left[\left(H_{s}^{\varepsilon_{n(m)}}\right)_{s>0} \in \cdot\right] \underset{m \rightarrow \infty}{\Longrightarrow} \mathbb{P}\left[\left(H_{s}\right)_{s>0} \in \cdot\right]
$$

proving (1.12).

2.3. Proof of the lemmas. In this section, we complete the proof of Theorem 1.2 by proving Lemmas 2.5-2.7.

Proof of Lemma 2.5: Since $F(t)$ is a continuous-time random walk on $\mathbb{Z}$ that jumps one step up or down with rate one each, the existence of a coupling such that (2.12) (i) holds follows by standard arguments, but to also get (2.12) (ii) we have to work a bit.

We observe from (2.11) and (2.8) that

$$
G^{(\varepsilon)}(s, t)=\varepsilon\left|\Pi_{\text {in }} \cap[-1,0 \wedge(1-2 \varepsilon s)] \times\left[-\frac{1}{2} \varepsilon^{-2} t, 0\right]\right| .
$$

Consider the map $\psi_{\varepsilon}: \mathbb{R}^{2} \rightarrow \mathbb{R}^{2}$ defined as

$$
\psi_{\varepsilon}(p, t):=\left((2 \varepsilon)^{-1}(1+p),-2 \varepsilon^{2} t\right),
$$

and let $\Xi^{\varepsilon}$ denote the random sum of delta measures

$$
\Xi^{\varepsilon}:=\sum_{(p, t) \in \Pi_{\mathrm{in}}} \delta_{\psi_{\varepsilon}(p, t)}
$$

Then $\Xi^{\varepsilon}$ is a Poisson point process on $\left(-\infty,(2 \varepsilon)^{-1}\right] \times \mathbb{R}$ with intensity $\varepsilon^{-1}$, and

$$
G^{(\varepsilon)}(s, t)=\varepsilon \int 1_{[0, s] \times[0, t]} \mathrm{d} \Xi^{\varepsilon}
$$

where $1_{A}$ denotes the indicator function of a set $A$. We can couple the processes $\Xi^{\varepsilon}$ for different values of $\varepsilon$ in a monotone way, i.e., such that processes with intensities $\varepsilon_{1}^{-1} \leq \varepsilon_{2}^{-2}$ satisfy $\Xi^{\varepsilon_{1}} \leq \Xi^{\varepsilon_{2}}$ a.s. (Note that also the domain $\left(-\infty,(2 \varepsilon)^{-1}\right] \times \mathbb{R}$ is a monotone function of $\varepsilon^{-1}$.) Setting up such a coupling in the obvious way, we will have that for each bounded measurable $A \subset \mathbb{R}^{2}$, the process $t \mapsto \Xi^{1 / t}(A)$ is (for large enough $t$ ) a standard Poisson process, i.e., a Markov process on $\mathbb{N}$ that jumps one step up with rate one and never jumps down. The strong law of large numbers then implies that for this sort of coupling,

$$
\varepsilon \Xi^{\varepsilon} \underset{\varepsilon \rightarrow 0}{\Longrightarrow} \ell \text { a.s. }
$$

where $\ell$ denotes the Lebesgue measure on $\mathbb{R}^{2}$ and $\Rightarrow$ denotes vague convergence of locally finite measures on $\mathbb{R}^{2}$.

We equip the space of all cadlag (right-continuous with left limits) functions $f:[0, \infty) \rightarrow \mathbb{R}$ with the Skorohod topology (see Kallenberg, 1997, Appendix A2) and we equip the space of all locally finite measures on $\mathbb{R}^{2}$ with the topology of vague convergence. Standard results show that with respect to the Skorohod topology, $F^{(\varepsilon)}=\left(F^{(\varepsilon)}(t)\right)_{t \geq 0}$ converges weakly in law to standard Brownian motion 
as $\varepsilon \downarrow 0$, while (2.30) shows that with respect to the topology of vague convergence, $\varepsilon \Xi^{\varepsilon}$ converges weakly in law to the deterministic limit $\ell$. We can now apply the Skorohod representation theorem Kallenberg (1997, Thm 3.30) to conclude that for each $\varepsilon_{n} \downarrow 0$, the random variables $\left(F^{\left(\varepsilon_{n}\right)}, \varepsilon_{n} \Xi^{\varepsilon_{n}}\right)$ can be coupled such that a.s.,

$$
F^{\left(\varepsilon_{n}\right)} \underset{n \rightarrow \infty}{\longrightarrow} B \text { and } \varepsilon_{n} \Xi^{\varepsilon_{n}} \underset{n \rightarrow \infty}{\longrightarrow} \ell,
$$

where $B=\left(B_{t}\right)_{t \geq 0}$ is a standard Brownian motion, $\rightarrow$ denotes convergence w.r.t. the Skorohod topology and $\Rightarrow$ denotes vague convergence of locally finite measures on $\mathbb{R}^{2}$.

Since $B$ has continuous sample paths, the a.s. convergence of $F^{\left(\varepsilon_{n}\right)}$ to $B$ in the Skorohod topology is equivalent to locally uniform convergence, which gives us (2.12) (i). Since the indicator functions $1_{[0, s] \times[0, t]}$ are a.e. continuous with respect to Lebesgue measure, by (2.29), the a.s. vague convergence of $\varepsilon_{n} \Xi^{\varepsilon_{n}}$ to $\ell$ implies the a.s. pointwise convergence

$$
G^{\left(\varepsilon_{n}\right)}(s, t) \underset{n \rightarrow \infty}{\longrightarrow} s t \quad(s, t \geq 0) .
$$

Since $G^{\left(\varepsilon_{n}\right)}$ is a.s. monotone in both $s$ and $t$, Lemma 2.8 below allows us to conclude that this convergence must in fact be uniform on rectangles of the form $[0, S] \times[0, T]$ as in (2.12) (ii).

Lemma 2.8 (Convergence of monotone functions). Let $C:=I_{1} \times \cdots \times I_{d}$ be $a$ hypercube in $[-\infty, \infty]^{d}$, where $I_{i}=\left[I_{i}^{-}, I_{i}^{+}\right]$is a compact interval for each $i=$ $1, \ldots, d$. Denote elements of $C$ as $x=\left(x_{1}, \ldots, x_{d}\right)$ and equip $R$ with the partial order $x \leq y$ iff $x_{i} \leq y_{i}$ for all $i=1, \ldots, d$. Let $g_{n}, g$ be real functions such that $\lim _{n \rightarrow \infty} g_{n}(x)=g(x)$ for all $x \in C$. Assume that each $g_{n}$ is monotone in the sense that $x \leq y$ implies $g_{n}(x) \leq g_{n}(y)$. Then

$$
\sup _{x \in C}\left|g_{n}(x)-g(x)\right| \underset{n \rightarrow \infty}{\longrightarrow} 0 \text {. }
$$

Proof: We first prove the statement for $C \subset \mathbb{R}^{d}$. Assume that (2.33) does not hold. Then we can find $\varepsilon>0$ and $x(n) \in C$ such that $\left|g_{n}(x(n))-g(x(n))\right| \geq \varepsilon$ for all $n$. By the compactness of $C$, going to a subsequence if necessary, we can assume that $x(n) \rightarrow x$ for some $x \in C$. Let

$$
K_{\delta}(x):=C \cap\left\{y \in \mathbb{R}^{d}:\left|x_{i}-y_{i}\right| \leq \delta \forall i=1, \ldots, d\right\} .
$$

By the continuity of $g$, we can find $\delta>0$ such that $|g(y)-g(x)| \leq \frac{1}{2} \varepsilon$ for all $y \in$ $K_{\delta}(x)$. Since $K_{\delta}(x)$ is the intersection of two hypercubes, it is itself a hypercube, i.e., there exist $x^{-}$and $x^{+}$such that

$$
K_{\delta}(x)=\left\{y \in \mathbb{R}^{d}: x^{-} \leq y \leq x^{+}\right\} .
$$

Since $x(n) \rightarrow x$, we have $x(n) \in K_{\delta}(x)$ for all $n$ large enough, and the monotonicity of $g_{n}$ then implies that

$$
g_{n}\left(x^{-}\right) \leq g_{n}(x(n)) \leq g_{n}\left(x^{+}\right) .
$$

Letting $n \rightarrow \infty$, using the pointwise convergence of $g_{n}$ to $g$, we see that

$$
\begin{aligned}
& g(x)-\frac{1}{2} \varepsilon \leq g\left(x^{-}\right) \leq \liminf _{n \rightarrow \infty} g_{n}(x(n)) \\
& \quad \leq \limsup _{n \rightarrow \infty} g_{n}(x(n)) \leq g\left(x^{+}\right) \leq g\left(x^{+}\right)+\frac{1}{2} \varepsilon,
\end{aligned}
$$

which contradicts the assumption that $\left|g_{n}(x(n))-g(x(n))\right| \geq \varepsilon$ for all $n$. 
This concludes the proof when $C \subset \mathbb{R}^{d}$. The more general case $C \subset[-\infty, \infty]^{d}$ is not really more general since $[-\infty, \infty]^{d}$ is isomorphic to $[0,1]^{d}$, both in the sense of topology and in the sense of the partial order $\leq$.

Proof of Lemma 2.6: Since $G^{(\varepsilon)}(s, t)$ is a.s. nondecreasing as a function of $s$, it suffices to prove the statement for $s$ sufficiently small; in particular, we can assume without loss of generality that $s \in\left(0, \frac{1}{2}\right]$. Then, for each $\varepsilon \in(0,1]$, by $(2.11)$,

$$
F^{(\varepsilon)}(t)-G^{(\varepsilon)}(s, t)=\varepsilon F\left(\frac{1}{2} \varepsilon^{-2} t\right)-\varepsilon G^{2 \varepsilon s}\left(\frac{1}{2} \varepsilon^{-2} t\right) \quad(t \geq 0),
$$

so we can rewrite $(2.13)$ as

$$
\lim _{T \rightarrow \infty} \sup _{\varepsilon \in(0,1]} \mathbb{P}\left[F(t)-G^{2 \varepsilon s}(t) \geq 0 \text { for some } t \geq \frac{1}{2} \varepsilon^{-2} T\right]=0 .
$$

Using notation as in (2.4), this says that

$$
\lim _{T \rightarrow \infty} \sup _{\varepsilon \in(0,1]} \mathbb{P}\left[E^{2 \varepsilon s}(t) \leq 0 \text { for some } t \geq \frac{1}{2} \varepsilon^{-2} T\right]=0 .
$$

Setting $\delta:=2 \varepsilon s$, this says that

$$
\lim _{T \rightarrow \infty} \sup _{\delta \in(0,2 s]} \mathbb{P}\left[E^{\delta}(t) \leq 0 \text { for some } t \geq 2 s^{2} \delta^{-2} T\right]=0 .
$$

Since this should hold for any $s \in\left(0, \frac{1}{2}\right]$, it suffices to show that

$$
\lim _{T \rightarrow \infty} \sup _{\delta \in(0,1]} \mathbb{P}\left[E^{\delta}(t) \leq 0 \text { for some } t \geq \delta^{-2} T\right]=0,
$$

where $\left(E^{\delta}(t)\right)_{t \geq 0}$ is a continuous-time random walk on $\mathbb{Z}$ that starts in $E^{\delta}(0)=0$ and jumps up by one with rate 1 and down by one with rate $1-\delta$.

Using the well-known fact that

$$
\mathbb{P}\left[E^{\delta}(t) \leq 0 \text { for some } t \geq 0 \mid E^{\delta}(0)=x\right]=1 \wedge(1-\delta)^{x}
$$

and the Markov property, it suffices to show that

$$
\lim _{T \rightarrow \infty} \sup _{\delta \in(0,1]} \mathbb{E}\left[1 \wedge(1-\delta)^{E^{\delta}\left(\delta^{-2} T\right)}\right]=0 .
$$

Since $E^{\delta}(t)$ is the difference of two independent Poisson distributed random variables with mean $t$ and $(1-\delta) t$, respectively,

$$
\mathbb{E}\left[E^{\delta}\left(\delta^{-2} T\right)\right]=\delta^{-1} T \text { and } \operatorname{Var}\left(E^{\delta}\left(\delta^{-2} T\right)\right)=(2-\delta) \delta^{-2} T
$$

Estimating $2-\delta \leq 2$, Chebyshev's inequality gives

$$
\mathbb{P}\left[\left|E^{\delta}\left(\delta^{-2} T\right)-\delta^{-1} T\right| \geq r \delta^{-1} \sqrt{2 T}\right] \leq r^{-2} \quad(r \geq 1) .
$$

It follows that

$$
\mathbb{E}\left[1 \wedge(1-\delta)^{E^{\delta}\left(\delta^{-2} T\right)}\right] \leq(1-\delta)^{\delta^{-1}(T-r \sqrt{2 T})}+r^{-2} \quad(r \geq 1) .
$$

Since $\log (1-\delta) \leq-\delta$ on $(0,1]$, we have

$$
(1-\delta)^{\delta^{-1}}=e^{\delta^{-1} \log (1-\delta)} \leq e^{-1} \quad(\delta \in(0,1])
$$

and hence

$$
\mathbb{E}\left[1 \wedge(1-\delta)^{E^{\delta}\left(\delta^{-2} T\right)}\right] \leq e^{-(T-r \sqrt{2 T})}+r^{-2} \quad(r \geq 1) .
$$

It follows that

$$
\limsup _{T \rightarrow \infty} \sup _{\delta \in(0,1]} \mathbb{E}\left[1 \wedge(1-\delta)^{E^{\delta}\left(\delta^{-2} T\right)}\right] \leq r^{-2} \quad(r \geq 1) .
$$


Since $r$ is arbitrary, this proves (2.44).

Proof of Lemma 2.7: Let $\mu[h]$ denote the locally finite measure on $(0, \infty]$ that $h \in$ $\mathcal{H}$ is the distribution function of. Then $\mu\left[h^{n}\right]$ converges vaguely to $\mu[h]$ if and only if $h_{s}^{n} \rightarrow h_{s}$ for each $s$ that is a point of continuity of the limiting function $h$. In particular, if $h$ is continuous, then $h^{n} \rightarrow h$ in the topology on $\mathcal{H}$ if and only if $h_{s}^{n} \rightarrow h_{s}$ for all $s \in(0, \infty)$, proving the equivalence of (i) and (iii). The implication (ii) $\Rightarrow$ (iii) is trivial while (iii) $\Rightarrow$ (ii) follows from Lemma 2.8 .

2.4. Ergodicity. In this section, we prove Proposition 1.1. Recall the definitions of $\underline{Y}^{\lambda}(t)$ and $\underline{N}^{\lambda}(t)$ from (2.3). Extending our definition in (2.1), for $\lambda \geq 0$, we define random maps $\left(\mathbf{Y}_{s, t}^{\lambda}\right)_{s \leq t}$ by

$$
\mathbf{Y}_{s, t}^{\lambda}(y):=Y(t) \cap[-\lambda, 0] \quad(t \geq 0)
$$

where $y$ is a finite subset of $[-\lambda, 0]$ and $(Y(t))_{t \geq s}$ is the inbox process started at time $s$ in an initial state with $Y(s) \cap[-\lambda, 0]=y$ and defined in terms of $\Pi_{\text {in }}$ and $\Pi_{\text {out }}$ as in Section 1.1. Note that by consistency, $Y(t) \cap[-\lambda, 0]$ is a function of $Y(s) \cap[-\lambda, 0]$ only.

Lemma 2.9 (Geometric distribution). One has

$$
\mathbb{P}\left[\underline{N}^{\lambda}(0)=n\right]=(1-\lambda) \lambda^{n} \quad(0 \leq \lambda<1, n \geq 0) .
$$

Proof: We have $\underline{N}^{\lambda}(0)=\left|\underline{Y}^{\lambda}(0)\right|$, where $\underline{Y}^{\lambda}(0)$ is the a.s. limit of $\mathbf{Y}_{-t, 0}^{\lambda}(\emptyset)$ as $t \rightarrow \infty$. Now $\left|\mathbf{Y}_{-t, s}^{\lambda}(\emptyset)\right|$, as a function of $s$, is a Markov process that jumps as in (1.5). It is easy to check that for $\lambda<1$, the geometric distribution with parameter $\lambda$ satisfies the detailed balance conditions and hence is an invariant law; this also proves positive recurrence. By irredicubility, this is the long-time limit law started from any initial state, and hence the law of $\left|\underline{Y}^{\lambda}(0)\right|$.

Lemma 2.10 (Successful coupling). For each $0 \leq \lambda<1$ and finite set $y \subset[-\lambda, 0]$, one has

$$
\lim _{t \rightarrow \infty} \mathbb{P}\left[\mathbf{Y}_{-t, 0}^{\lambda}(y)=\underline{Y}^{\lambda}(0)\right]=1 .
$$

Proof: Since $\underline{Y}^{\lambda}(0)$ is a.s. finite by Lemma 2.9, and is the a.s. limit of $\mathbf{Y}_{-t, 0}^{\lambda}(\emptyset)$ as $t \rightarrow \infty$ by its definition in (2.2), in order to prove (2.53), it suffices to show that

$$
\lim _{t \rightarrow \infty} \mathbb{P}\left[\mathbf{Y}_{-t, 0}^{\lambda}(y)=\mathbf{Y}_{-t, 0}^{\lambda}(\emptyset)\right]=1
$$

By monotonicity (Lemma 2.1), $\mathbf{Y}_{-t, s}^{\lambda}(y) \supset \mathbf{Y}_{-t, s}^{\lambda}(\emptyset)$ for all $s \in[-t, 0]$. In particular, if $\mathbf{Y}_{-t, s}^{\lambda}(y)=\emptyset$ for some $s \in[-t, 0]$, then $\mathbf{Y}_{-t, s}^{\lambda}(\emptyset)=\emptyset$ for the same $s$ and the two processes are equal at each later time, so

$$
\mathbb{P}\left[\mathbf{Y}_{-t, 0}^{\lambda}(y)=\mathbf{Y}_{-t, 0}^{\lambda}(\emptyset)\right] \geq \mathbb{P}\left[\mathbf{Y}_{-t, 0}^{\lambda}(y)=\emptyset \text { for some } s \in[-t, 0]\right] .
$$

Since $\left|\mathbf{Y}_{-t, s}^{\lambda}(y)\right|$, as a function of $s$, is a Markov process that jumps as in (1.5), which is recurrent for $\lambda \leq 1$, the right-hand side of (2.55) tends to one as $t \rightarrow \infty$.

Proof of Proposition 1.1: Defining $Y^{1}(\infty):=\underline{Y}^{1}(0)$, the convergence in total variation distance in (1.7) is an immediate consequence of the coupling in Lemma 2.10, while $N^{\lambda}(\infty)$ is geometrically distributed by Lemma 2.9. Since $\left|Y^{\lambda}(\infty)\right|$ is geometrically distributed with parameter $\lambda$, we see that $\left|Y^{\lambda}(\infty)\right|$ tends to infinity in probability as $\lambda \uparrow 1$. Since $Y^{\lambda}(\infty) \uparrow Y^{1}(\infty)$, this implies that $Y^{1}(\infty)$ is a.s. an infinite set. 


\section{Acknowledgments}

Work sponsored by GAČR grant P201/12/2613.

\section{References}

P. Bak and K. Sneppen. Punctuated equilibrium and criticality in a simple model of evolution. Phys. Rev. Lett. 71, 4083-4086 (1993). DOI: 10.1103/PhysRevLett.71.4083.

A.-L. Barabasi. The origin of bursts and heavy tails in human dynamics. Nature 435, 207-211 (2005). DOI: 10.1038/nature03459.

P. Blanchard and M.-O. Hongler. Modeling human activity in the spirit of barabasi's queueing systems. Phys. Rev. E 75, 026102 (2007). DOI: 10.1103/PhysRevE.75.026102.

E. Brunet, B. Derrida, A. H. Mueller and S. Munier. Noisy traveling waves: Effect of selection on genealogies. EPL (Europhysics Letters) 76 (1), 1 (2006).

M. Formentin, A. Lovison, A. Maritan and G. Zanzotto. Hidden scaling patterns and universality in written communication. Phys. Rev. E 90, 012817 (2014). DOI: 10.1103/PhysRevE.90.012817.

A . Gabrielli and G. Caldarelli. Invasion percolation and the time scaling behavior of a queuing model of human dynamics. J. of Stat. Mech.: Theory and Experiment 2009 (02), P02046 (2009).

P. Groeneboom. The concave majorant of Brownian motion. Ann. Probab. 11 (4), 1016-1027 (1983). MR714964.

O. Kallenberg. Foundations of modern probability. Probability and its Applications (New York). Springer-Verlag, New York (1997). MR1464694.

T. M. Liggett. Interacting particle systems. Classics in Mathematics. SpringerVerlag, Berlin (2005). ISBN 3-540-22617-6. Reprint of the 1985 original. MR2108619.

H. Luckock. A steady-state model of the continuous double auction. Quant. Finance 3 (5), 385-404 (2003). MR2015235.

P. Maillard. Speed and fluctuations of N-particle branching Brownian motion with spatial selection. Probab. Theory Related Fields 166 (3-4), 1061-1173 (2016). MR3568046.

R. Meester and A. Sarkar. Rigorous self-organised criticality in the modified BakSneppen model. J. Stat. Phys. 149 (5), 964-968 (2012). MR2999570.

G. J. Stigler. Public regulation of the securities markets. The Journal of Business 37 (2), 117-142 (1964).

J. M. Swart. A simple rank-based markov chain with self-organized criticality. ArXiv Mathematics e-prints (2015). arXiv: 1405.3609v3.

A. Vázquez, J. G. Oliveira, Z. Dezsö, K.-I. Goh, I. Kondor and A.-L. Barabási. Modeling bursts and heavy tails in human dynamics. Phys. Rev. E 73, 036127 (2006). DOI: 10.1103/PhysRevE.73.036127. 